\title{
Risk factors for sleep disturbance and its effect on quality of life: An Analysis of Retrospective Cohort Study of adults with neck injuries
}

\author{
Ahmed Hossain and Hafiz T. A. Khan \\ Department of Public Health Sciences, University of Toronto, 155 College Street, Toronto, Ontario M5T \\ $3 \mathrm{M7}$, Canada
}

\begin{abstract}
Indian J Sleep Med 2007; 2.3, 95-100

Introduction: Persons with neck injuries often have lasting psychosocial effects. One symptom that occurs in a significant number of persons is problems with sleep. Until recently, it was unclear about the predictors of sleep disturbances after a neck injury that does not tend to resolve within six months post injury. In addition, very little is known about how sleep affects overall self reported quality of life, controlling for other relevant predictors. The study aims to examine predictors of sleep disturbances after a neck injury that does not tend to resolve within six months of post injury and how sleep disturbance affects overall quality of life to those having six months post injuries.
\end{abstract}

Methods: Data from a retrospective cohort study of adults have been used to investigate the study. The study population were 258 adults aged (at least 14 years) suffering from moderate to severe neck injury at an acute care Hospital in Toronto, Canada. Both exploratory as well as advanced logistic regression analyses were used in the study.

Results: The study shows that participants who had alcohol problem are experiencing about two times higher sleep disturbance and participants with lower education are approximately 3 times more likely of having trouble with sleep than those with higher education. The injury severity and marital status are also found to be important determinant for sleep disturbances. Again, as expected, sleep disturbance and mental health are associated with defining quality of life.

Conclusion: This study reveals that marriage, alcohol problem, education, mental health and injury severity are significantly associated with sleep disturbance. On the other hand, strong associations are observed among sleep, mental health and quality of life. These finding helps in understanding risk factors related to sleep disturbance and their consequences on the quality of life.

Keywords: sleep disturbance, quality of life, neck injury, polytomous logistic regression.

\section{Introduction}

Sleep disruption has a profound effect on an individual person and on the society as a whole. ${ }^{(1-3)}$ Poor sleep quality can result in excessive daytime sleepiness and is associated with significant socioeconomic consequences such as loss of productivity and increased road traffic accidents.

Address for Correspondence

Dr. Ahmed Hossain

Department of Public Health Sciences, University of Toronto, 155 College Street,

Toronto, Ontario M5T 3M7, Canada
Age is thought to be a higher night-time accident rate and thus young people are frequently involved in sleeprelated accidents compared to older counterparts. ${ }^{(4,5)}$ Epidemiological studies from other countries have shown a high prevalence of sleep complaints, including snoring, insomnia and excessive daytime sleepiness. ${ }^{(6-9)}$

Past studies have attempted to examine the sleep disorders of population suffering from certain disease and explored its impact on the quality of life (QoL). ${ }^{(10)}$ Williams et al. have shown that a high prevalence of sleep problems reported by parents of children with autism. ${ }^{(1)}$ Disturbed sleep has been linked to impaired 
vigilance, learning memory and creative thought in numerous studies. ${ }^{(12,13)}$ The sleep disorder inventory describes the frequency, severity and caregiver burden of sleep-disturbed. ${ }^{(14,15)}$ Sleep disturbances may contribute to the behavioral, functional, and cognitive status of persons with Alzheimer's disease as well as to burden and health status of the caregiver. ${ }^{(15-17)}$

Sleep is generally assumed to be conducive to healing. "From a clinical perspective, sleep disruption frequently occurs postoperatively or following an acute injury and could thus affect wound healing”. ${ }^{(18)}$ Sleep complaints and disorders are increasingly reported by patients to physicians now-a-days. ${ }^{(19-21)}$ Researchers have sought to find explanation of sleep disturbance for many years. Neck injury or other kind of injuries could be some of the most important risk factors for sleep disturbance. Sleep loss, insufficient sleep during the night or having poor sleep habits may affect in perceiving a better QoL. Thereby, this study aims to elucidated whether associations between sleep disturbance and QoL exits, and if present, how. It is also important to provide further information on how socio-demographic factors and injury related factors affect patients' sleep behavior. Therefore, it is important to know the association of daily sleep habits with the pre-injury socio-demographic factors and injury related factors and their effect on the overall QoL. Over the past few years considerable interest has centered on assessing QoL in patients with obstructive sleep apnea. ${ }^{(3,22-25)}$ These studies have been carried out in the past despite there is ongoing debates as to exactly what measures should be used to characterize sleep and how to measure the QoL. ${ }^{(10,26-29)}$

In the present study, we conducted a retrospective cohort study of 258 adults with moderate to severe neck injuries from an acute care hospital in Toronto. Data on age, gender, marital status, education at time of injury, prior alcohol problems and three other injury related factors like severity score of neck injury (NeckSev), overall injury severity score (Iss) and length of hospital stay (lnSty) were collected in this study. Also physical function (physF), trial making test A (trla), trial making test B (TrlB), mental health (MenHlt), length of followup(lngFll), tiredness (tired), trouble with sleep (sleep) and self reported instrument of quality of life (QoL) were collected during post injury period in this study. At first, we analyze to identify the potential risk factors for sleep disturbance by taking the socio-demographic factors and injury related factors into account. Then, we investigate the hypothesis that troubles with sleep are important risk factors for QoL. We analyze participants with poor sleep habits and participants with better sleep habits individually in addition to both combined, since participants trouble with sleep are reported to have a higher incidence of having poor QoL compared to participants with moderate and highest QoL.

\section{Methods}

This study involved analysis of data from a retrospective cohort study of adults $(n=258)$ with moderate to severe neck injuries from an acute care hospital in Toronto, Canada. A consecutive series of eligible participants who were discharged from an acute care hospital were traced and later interviewed. Participants had to be at least 14 years of age at the time of injury and live within a 100 mile radius catchments area from the project office in Toronto. The majority of participants were interviewed in their home. Participants were also asked to provide consent to abstract medical records for information as well such as injury severity. Data on age, gender, marital status, education at time of injury and pre-injury behavioral problems were abstracted from the medical records. Information related to prior alcohol and/or substance abuse problems, prior problems with the law, and prior psychiatric problems was collapsed into two categories: no problems with alcohol, controlled substances, the law, or psychiatric issues were coded as 1 (that is, if the subject had a problem with any of those issues at the time of the accident, then the variable was coded as one).

Severity of neck injury was based on a nine level ordinal scale. A higher score indicated more injury severity. The Injury Severity Score (Iss) was derived from ICD-9 codes of hospital discharge abstracts. This score indicates the severity of injury based on the number of other injuries occurred including the neck injury and could range from $0-75$, with a higher score indicating more severity. Length of hospital stay was measured in days and calculated based on recorded admission and discharge dates from the hospital.

The Physical Function subscale of the Medical Outcomes Study Short Form 36 (SF-36) was used as a measure of physical status at the time of follow-up. The Physical Function subscale consists of 10-items (e.g. walking, climbing stairs, and lifting heavy objects). The subscales are scored by summing the raw scale scores and transforming raw scores to a 0 to 100 scale (higher 
scores indicate higher levels of physical function). The Mental Health subscale of the SF-36 was used as a measure of affective status at the time of follow-up. This subscale consists of five items, which are intended to reflect emotional or feeling states (e.g. feeling depressed, worn-out, tired). Scores range from 0 to 100 in which higher score indicates higher levels of emotional wellbeing.

A cognitive battery was administered at the time of follow-up and included the Trail Making Test (TMT), Parts A and B. The TMT tests attention, sequencing, mental flexibility, visual search, and motor function. A lower score on this test indicated fewer cognitive problems.

Trouble with sleep was dichotomized and coded as 1 if this was positive. Perceived QoL is a self report instrument. It is measured on an 11 point scale where 0 represented the lowest QoL and 10 indicated the highest QoL. We then subsequently define the QoL in 3 categories: poor (scale value, $0-3$ ), moderate (scale value, 4-7) and highest (scale value, $8+$ ).

Associations of trouble with sleep and pre-injury socio-demographic as well as injury and post injury related factors are identified with a series of multivariable logistic regression analyses. Scatter plots were created to visually examine the relationship among the variables. Fig. 1 and Fig. 2 show the scatter plots for injury related factors and post injury related factors respectively. It is apparent from the Fig. 1 that there is strong association exists among injury related factors which will be the cause of colinearity and it will give less efficient results. For this reason and to avoid colinearity in the model, we only evaluate the overall injury severity score (ISS). We evaluate the trouble with sleep by ISS, Physical function and mental health in the presence of some potential confounding factors complaint by age, sex, alcohol problem, education level in the logistic regression models.

A strong correlation is apparent between trial marking test A (trla) and trial marking test B ( TrlB) from the Fig. 2. We, therefore, postulate an interaction effect of trla and TrlB present to evaluate QoL. We analyze participants with poor sleep habits and participants with better sleep habits individually since participants trouble with sleep are expected to have a higher incidence of having poor QoL compared to participants with highest QoL. It will also help to avoid sleep disturbance as a potential confounder in the model to interpret QoL. At last, we used a stepwise procedure for a model selection including all socio-demographic factors, pre injury related factors and post-injury related factors in the model. The data were analyzed by the Statistical Package for the SAS system $9.1 .^{(30)}$

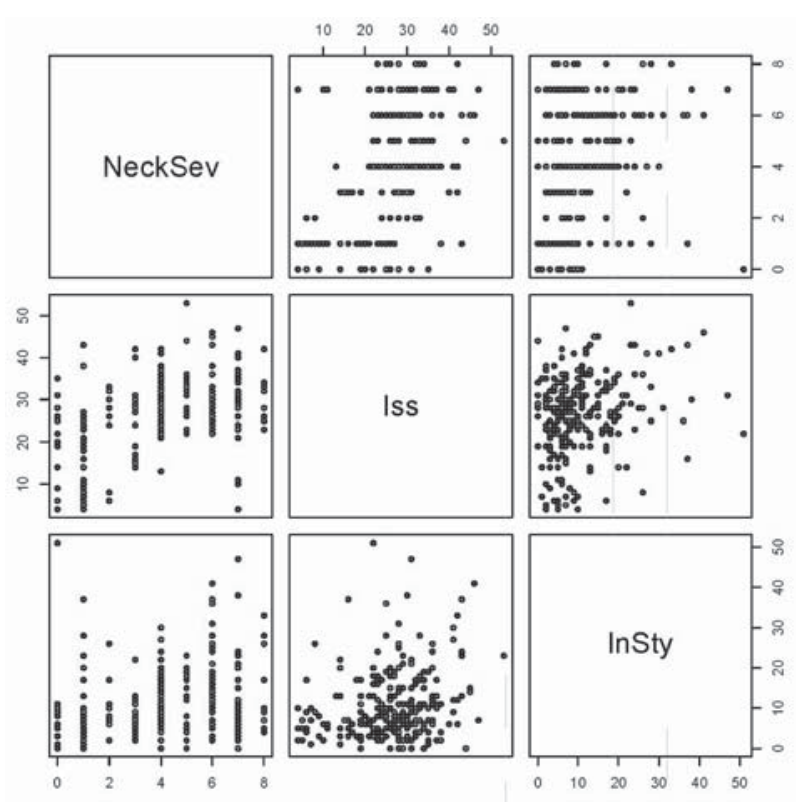

Fig 1: Scatter plots for Injury-related Factors: red color represents subjects have had no sleep difficulty and green color represents subjects have had sleep difficulty.

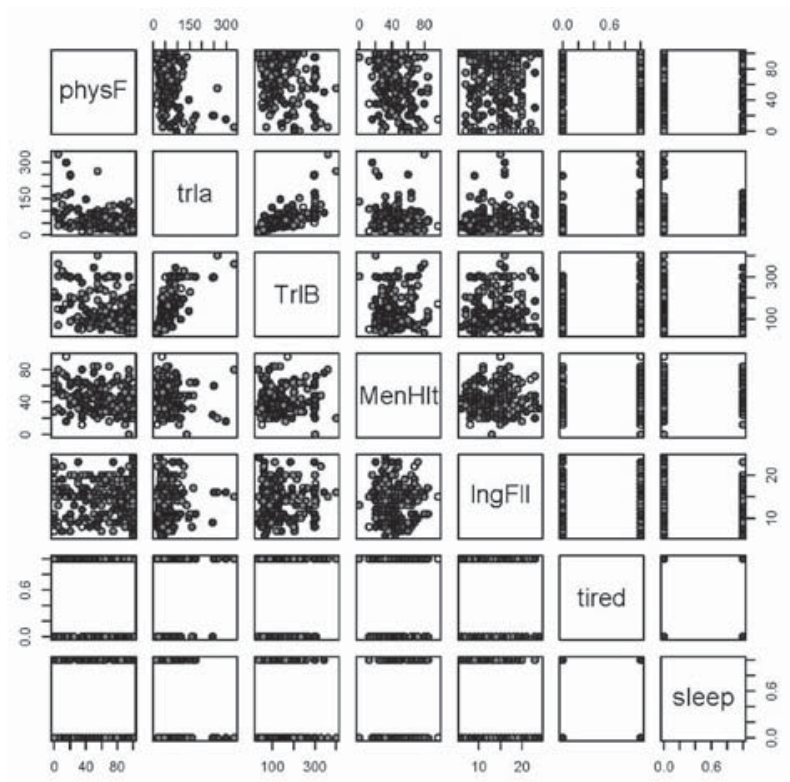

Fig 2: Scatter plots for Post Injury Personal Factors: red color represents subject has lower QoL, green color represents moderate QoL and blue color represents with highest QoL.

Indian Journal of Sleep Medicine (IJSM), Vol. 2, No. 3, 2007 


\section{Results}

The age of participants was at least 14 years. About $62 \%$ of participants were between 14 and 29 years in age. Among these young participants, about $21 \%$ had sleep problems. Sixty-two percent of participants were females and among them $25 \%$ had sleeping difficulties. Education of the participants was measured according to their completed years of schooling. As concerning education, more than $27 \%$ of all participants went for higher education (12+ years of schooling) and among them more than $22 \%$ had trouble with sleep. On the whole nearly $47 \%$ participants of the study complaint sleep difficulties. A brief summary of the demographic and injury related factors with sleep complaints of the study population has been given in Table I.

It is found that the mean (standard deviation) of Iss and length of hospital stay (lnSty) is 26.25(9.49) and $10.77(8.59)$, respectively. In addition, the mean score of physical function of the participants is 65.83 with standard deviation 33.04. It is also found that $22.22 \%$ of the participants had trouble with sleep and among them $15.52 \%$ and $50 \%$ are leading poor and moderate QoL, respectively. It is also found that about $60 \%$ of the participants who didn't complain about sleep perceive highest QoL.

Table 1: Summary of socio-demographics and neck injury severity of the study population.

\begin{tabular}{|l|l|c|c|c|c|}
\hline \multirow{2}{*}{ Characteristics } & \multirow{2}{*}{ Measurement } & \multicolumn{2}{|c|}{ Patients } & \multicolumn{2}{c|}{ Slccp complaints } \\
\cline { 3 - 6 } & & $\mathrm{N}$ & $\%$ & $\mathrm{~N}$ & $\%$ \\
\hline Alcohol problem & Yes & 63 & 24.42 & 21 & 20.51 \\
& No & 195 & 75.58 & 40 & 33.33 \\
\hline Age (years) & $14-29$ & 159 & 61.63 & 33 & 20.75 \\
& $30-39$ & 44 & 17.05 & 14 & 31.82 \\
& $40-49$ & 29 & 11.24 & 7 & 24.14 \\
& $50-59$ & 18 & 6.98 & 6 & 33.33 \\
& $60+$ & 8 & 3.10 & 1 & 12.50 \\
\hline Marital status & Married & 97 & 37.60 & 32 & 33.99 \\
& Single & 161 & 62.40 & 29 & 18.01 \\
\hline Gender & Female & 160 & 62.02 & 40 & 25.00 \\
& Malc & 98 & 37.98 & 21 & 21.43 \\
\hline Fducation & $1-9$ & 19 & 7.69 & 7 & 36.84 \\
(years of schooling) & $10-12$ & 161 & 65.18 & 39 & 24.22 \\
& $12+$ & 67 & 27.13 & 15 & 22.39 \\
\hline Neck injury severity & Moderate (0-4) & 152 & 58.91 & 37 & 24.34 \\
& Severe (5+) & 106 & 41.09 & 24 & 22.64 \\
\hline
\end{tabular}

We examine the associations of trouble with sleep and pre-injury socio-demographic as well as injury and post injury related factors are examined by fitting a multivariable logistic regression model. The decision to remove scores of tiredness is made based on high biological association with physical function and mental health. The logistic regression analysis indicates that participants with alcohol problem, lower education, single participants, lower score of mental health and lower Iss have significantly higher prevalence of sleep disturbance (Table II). The '*' signs in Table II indicates lower p-value of the estimates and proofs the significance of the estimates at $10 \%$ significance level.

The coefficients in logistic regression are given in terms of the log odds. We can express the coefficient in odds by getting rid of the $\log$ by taking e to the power for both sides of the equation. The coefficient for alcohol problem (No) -0.3876 implies that the participants who didn't have alcohol problem are $\left(\mathrm{e}^{-0.3876}\right) 0.68$ times lower of having sleep disturbance than who had alcohol problem. Again the coefficient for Iss 0.0407 implies that a one unit change in injury severity score results in $\mathrm{a}\left(\mathrm{e}^{0.0407}=\right) 1.05$ unit change in the odds. It is also apparent from Table II that the participants who were single have 2.054 times higher sleep disturbance than who were married. Participants with 1 grade lower in education are found to be approximately 1.2 times more likely of having trouble with sleep. Mental health is found to be the most significant predicator for sleep disturbance. In addition, the variables age, sex, and physical function are found these variables don't have any significant effect on sleep disturbance.

Table 2: Analysis of maximum likelihood estimates for sleep predictors

\begin{tabular}{|l|l|l|l|l|}
\hline Parameter & Estimate & SE & Wald $\chi^{2}$ & p-value \\
\hline Intercept & 1.6295 & 0.7919 & 4.2342 & 0.0396 \\
\hline $\begin{array}{l}\text { Alcohol } \\
\text { problem (No) }\end{array}$ & -0.3876 & 0.1801 & 4.6335 & $0.0314^{* *}$ \\
\hline Age & -0.0092 & 0.0175 & 0.2768 & 0.5988 \\
\hline $\begin{array}{l}\text { Marital status } \\
\text { (Unmarried) }\end{array}$ & -0.4338 & 0.2118 & 4.1938 & $0.0406^{* *}$ \\
\hline $\begin{array}{l}\text { Gender } \\
\text { (Female) }\end{array}$ & -0.0433 & 0.1762 & 0.0603 & 0.8061 \\
\hline Education & -0.1766 & 0.0978 & 3.2602 & $0.0710^{*}$ \\
\hline Injury severity & 0.0407 & 0.0188 & 4.6800 & $0.0305^{* *}$ \\
\hline $\begin{array}{l}\text { Physical } \\
\text { Function }\end{array}$ & 0.0005 & 0.0060 & 0.0071 & 0.9330 \\
\hline Mental Health & 0.0300 & 0.0113 & 7.0897 & $0.0078^{* * *}$ \\
\hline
\end{tabular}

Note: Significant at ${ }^{*} \mathrm{p}<0.10 ;{ }^{* *} \mathrm{p}<0.05 ;{ }^{* * *} \mathrm{p}<0.01$

The dependent variable, QoL, has 3 levels representative of poor, moderate and highest QoL and for this we carry out a polytomous logistic regression analysis to evaluate the QoL. The participants with poor sleep habits (sleep $=0$ ) and participants with better sleep habits ( sleep $=1)$ are analyzed individually since 
participants who trouble with sleep are expected to have a higher incidence of having poor QoL compared to participants with highest QoL. And by analyzing individually we can avoid sleep disturbance as a potential confounder in the model. We added an interaction effect $\operatorname{TrlA}$ and TrlB in the model. The results of the odds ratios are displayed in Table III. The results show that the odds ratio for variables such as marital status, sex, and education in completed years are significantly different for the two groups. The odds ratios of other variables also show some difference but all the variables include 1 in the confidence intervals of odds ratio except the mental health. Therefore, mental health has a significant effect on perceiving better QoL.

The polytomous logistic regression analysis including mental health, marital status, education and injury severity score have significant influence on perceiving better QoL. Finally, an odds ratio estimate is calculated with sleep variable only after running a polytomous logistic regression for QoL. It is found that participants with trouble with sleep perceiving 2.75 times lower QoL than who didn't have any sleep complaints.

Table 3: Odds ratio estimate (95 percent confidence interval) for quality of life predictors with separating two sleep complaint groups.

\begin{tabular}{|l|c|c|c|}
\hline Effect & Category & Sleep $=0$ & Sleep $=1$ \\
\hline $\begin{array}{l}\text { Alcohol } \\
\text { problem }\end{array}$ & $\begin{array}{c}\text { Yes vs } \\
\text { No }\end{array}$ & $1.58(0.62,4.05)$ & $1.76(0.52,5.95)$ \\
\hline Age & & $0.98(0.94,1.02)$ & $0.95(0.89,1.03)$ \\
\hline Marital starus & $\begin{array}{c}\text { Married } \\
\text { vs Single }\end{array}$ & $1.52(0.54,4.22)$ & $0.33(0.05,1.99)$ \\
\hline Gender & $\begin{array}{c}\text { Male vs } \\
\text { Femalc }\end{array}$ & $1.16(0.53,2.55)$ & $3.64(0.82,16.13)$ \\
\hline Education & & $0.83(0.66,1.03)$ & $1.18(0.67,2.08)$ \\
\hline Injury severity & & $0.99(0.95,1.03)$ & $0.96(0.89,1.04)$ \\
\hline $\begin{array}{l}\text { Physical } \\
\text { function }\end{array}$ & & $0.99(0.98,1.01)$ & $1.01(0.98,1.03)$ \\
\hline Mental health* & & $1.06(1.03,1.09)$ & $1.05(1.01,1.10)$ \\
\hline
\end{tabular}

\section{Discussion}

The study has several strengths, including wellcharacterized population and specificity of sleep complaints surveyed. Trouble with sleep was reported by $25.22 \%$ of the participants in the study. The relationship between sleep and aging in this study is quite complex because around $79 \%$ of the participants are between 14 years and 39 years of age; most of them belonging to the young population. However, sleep complaints are generally reported by elderly people and hence might contribute to ageing. ${ }^{(21,31-32)}$ Our study, which controlled for neck injured participants, suggests that it is not age per se that result in increasing sleep complaints, but surprisingly enough, marriage, alcoholism, and injury severity are amongst the factors related to sleep disturbance. Surprisingly higher educated participants reported to have less sleep complaints. Mental health is found an obvious risk factor for sleep disturbance. Again sex, age, physical function do not affect sleep disturbance.

The second part of the study involves finding the predictors for achieving better QoL. Among all post injury factors, mental health and sleep seem to play a significant role related to perceiving highest QoL. We analyze participants with poor sleep and participants with no sleep complaints individually. In addition, to know the sleep effect on QoL, we perform a polytomous logistic regression analysis with treating sleep as only predictor for defining QoL. It is expected with the findings that sleep complaints are reported to have a higher incidence of having poor QoL compared to participants with highest QoL. All the analysis show that mental health and sleep disturbance are the most consistent and robust predictor for the defining QoL which is in fact consistent with several previous studies. ${ }^{(19,25)}$ Physical function or cogitative problems were not found to be related to perceiving highest QoL. These findings may help clinicians evaluate patients with sleep complaints and those who do not perceive better QoL.

We have opted to assess the predictors and correlate sleep complaints with pre-injury socio-demographic factors besides injury related factors. In addition, we also took into account factors related to perceiving better quality of life. This study reveals that marriage, alcohol problem, education, mental health and injury severity are significantly associated with sleep disturbance. Strong associations are also observed among sleep, mental health and quality of life. These findings certainly add knowledge in understanding risk factors related to sleep disturbance and their consequences on the quality of life. Factors that appeared to be statistically insignificant need to be explored further.

\section{Acknowledgements}

The authors would like to thank Ms Shahnaz F. Haque, University of Sheffield for her meticulous proof reading. Of course, all remaining errors are the authors' own responsibility.

Indian Journal of Sleep Medicine (IJSM), Vol. 2, No. 3, 2007 


\section{References}

1. Shapiro CM, Dement WC. Impact and epidemiology of sleep disorders. B.M.J. 1993, 306:1604-7.

2. Nugent AM, Gleadhill I, Mccrum E, et al. Sleep complaints and risk factors for excessive daytime sleepiness in adult males in Northern Ireland. J. Sleep Res. 2001, 10:69-74.

3. Daniels E, King MA, Smith IE, Shneerson JM. Health-related quality of life in narcolepsy. J. Sleep Res. 2001, 10:75-81.

4. Åkerstedt $\mathrm{T}$, Kecklund $\mathrm{G}$. Age, gender and early morning highway accidents. J. Sleep Res. 2001, 10:105-10.

5. Philip P, Taillard J, Sagaspe P, et al. Age, performance and sleep deprivation. J. Sleep Res. 2004, 13:105-110.

6. Bixler EO, Kales A, Soldatos CR, Kales J, Healey S. Prevalence of sleep disorders in the Los Angeles metropolitan area. Am. J. Psych., 1979, 136:1257-62.

7. Klink M, Quan SF. Prevalence of reported sleep disturbances in a general adult population and their relationship to obstructive airways diseases. Chest, 1987, 91:540-6.

8. Gislason T, Almqvist M. Somatic diseases and sleep complaints. Acta Med. Scand. 1987, 221:475-81.

9. Stradling JR, Crosby JH, Payne CD. Self reported snoring and daytime sleepiness in men aged $35-65$ years. Thorax, 1991, 46:807-10.

10. Rao A, Georgiadou P, Francis DP, et al. Sleep-disordered breathing in a general heart failure population: relationships to neurohumoral activation and subjective symptoms. J. Sleep Res. 2006, 15:81-8.

11. Williams PG, Sears LL, Allard AM. Sleep problems in children with autism. J. Sleep Res. 2004, 13:265-8.

12. Gozal D. Sleep disordered breathing and school performance in children, pediatrics, 1998, 102:616-20.

13. Horne JA. Sleep loss and divergent thinking ability, Sleep, 1988, 11:528-36.

14. Yassouridis A, Steiger A, Klinger A, Fahrmeir L. Modelling and exploring human sleep with event history analysis. J. Sleep Res. 1999, 8:25-36.

15. Tractenberg $\mathbf{R}$, Singer $C M$, Cummings JL, Thal LJ. The sleep disorders inventory: an instrument for studies of sleep disturbance in persons with Alzheimer's disease, J. Sleep Res. 2003, 12:331-7.

16. Pollak CP, Perlick D. Sleep problems and institutionalization of the elderly. J. Geriatr. Psychiatry Neurol. 1991, 4:20410.

17. Pollak CP, Stokes PE. Circadian rest-activity rhythms in demented and nondemented older community residents and their caregivers. J. Am. Geriatr. Soc. 1997, 45:44652.

Indian Journal of Sleep Medicine (IJSM), Vol. 2, No. 3, 2007
18. Mostaghimi L, Obermeyer WH, Ballamudi B, MartinezGonzalez, Benca RM. Effects of sleep deprivation on wound healing. J. Sleep Res. 2005, 14:213-9.

19. Ancoli-Israel S. Epidemiology of sleep disorders. Clinical Geriatr Med, 1989, 5:347-62.

20. Camhi SL, Morgan WJ, Pernisco N, Quan SF. Factors affecting sleep disturbances in children and adolescents. Sleep Med 2000, 1: 117- 23.

21. Doghramii PP. Recognizing sleep disorders in a primary care setting. J Clin Psychiatry Supplementary 2004, 16:236.

22. Walker-Engström ML, Wilhelmsson B, Telgelberg $\AA$, Dimenäs $E$, Ringqvist I. Quality of life assessment of treatment with dental appliance or UPPP in patients with mild to moderate obstructive sleep apnoea. A prospective randomized 1 -year follow-up study.

23. Gordo MA, Recio J, Sanchez-Barcelo EJ. Decreased sleep quality in patients suffering from retinitis pigmentosa, $J$. Sleep Res. 2001, 10:159-64.

24. Moore P, Bardwell WA., Ancoli-Israel S, Dimsdale JE. Association between polysomnographic sleep measures and health-related quality of life in obstructive sleep apnea. J. Sleep Res. 2001, 10:303-8.

25. Phillips B, Mannino D. Correlates of sleep complaints in adults: The ARIC Study. J Clin Sleep Med 2005, 1:277-83.

26. Gehrman $P$, Matt GE, Turingan $M$, Dinh $Q$, Ancoli-Israel $S$. Towards an understanding of self-reports of sleep, J. Sleep Res. 2002, 11:229-36.

27. Ware JE Jr, Sherbourne CD. The MOS 36-item short-form health survey (SF_36) conceptual framework and item selection. Med. Care, 1992, 30:473-83.

28. Parker SG, Peet SM, Jagger C, Farhan M, Castleden CM. Measuring health status in older patients. The SF-36 in practice. Age Ageing, 1998, 27:13-8.

29. Berry C, McMurray JA. A review of quality of life evaluations in patients with congestive heart failure. Pharmacoeconomics, 1999, 16:247-71.

30. SAS 9.1 for Windows, SAS Institute Inc, Cary, NC, USA, 2003.

31. Hetta J, Broman JE, Mallon L. Evaluation of severe insomnia in the general population-implications for the management of insomnia: insomnia, quality of life, and healthcare consumption in Sweden. J Psychopharmacol 1999, 13:S356.

32. Namen AM, Wymer A, Case D, Haponik EF. Performance of sleep histories in an ambulatory medicine clinic: impact of simple chart reminders. Chest 1999, 116:1558-63. 